\title{
Perceived Depth Inversion of Smoothly Curved Surfaces Due to Image Orientation
}

\author{
Francene D. Reichel \\ Brandeis University
}

\author{
James T. Todd \\ Brandeis University \\ and Center for Adaptive Systems, Boston University
}

\begin{abstract}
A relative depth judgment task was used to distinguish perceived reversals in depth due to image orientation from spontaneous reversals such as those observed with a Necker cube. Results demonstrate that inversion effects due to image orientation can occur for several different types of pictorial representation and that many of these effects are incompatible with traditional explanations involving a perceptual bias for overhead illumination. When this bias was neutralized by placing the light source at the point of observation, the effects of image orientation were just as large as with overhead illumination. Similar results were also obtained for surfaces depicted with texture or motion in which all relevant shading information was eliminated. These results can be explained by a perceptual bias for backward slanting surfaces, but additional evidence suggests that this bias can be attenuated by the presence of smooth occlusion contours.
\end{abstract}

The phenomenon of shape constancy is a remarkable property of visual perception through which an object's structure can remain perceptually invariant over large changes in viewing perspective. There are, however, a variety of situations in which shape constancy breaks down. One particularly compelling example is demonstrated in Figure 1, which shows two identical shaded images of a smoothly curved surface in different orientations. Note in the figure that the surfaces are perceptually quite different: The one in the upper image appears to have a convex protuberance at its center, whereas the one in the lower image appears to have a concave depression. This striking perceptual distinction is due solely to image orientation. Indeed, if the entire figure is turned upside down, the perceived relief of each of the two surfaces is reversed.

It is important to note while considering Figure 1 that the depicted structure is mathematically ambiguous, much like the ambiguous structure of a Necker cube. There is an important phenomenological difference, however. Unlike a Necker cube, which appears to invert spontaneously in depth over time, the surface in Figure 1 tends to remain perceptually stable over continuous viewing within a given orientation of the image. It appears to reverse in depth only when the image is turned upside down.

One widely accepted hypothesis to account for depth inversion due to image rotation is that perceivers are biased to expect overhead illumination (e.g., see Benson \& Yonas,

This research was supported in part by the Air Force Office of Scientific Research (Grant 89-0016) and by a joint grant from the Office of Naval Research and the National Science Foundation (BNS8420143). The participation of Francene D. Reichel was also supported under a National Science Foundation Graduate Fellowship.

We are most grateful to Kevin Berbaum and James Cutting for their helpful comments on an earlier draft of the manuscript of this article.

Correspondence concerning this article should be addressed to Francene D. Reichel or James T. Todd, Department of Psychology, Brandeis University, Waltham, Massachusetts 02254.
1973; Berbaum, Bever, \& Chung, 1983, 1984; Brewster, 1826; Gibson, 1950; Hagen, 1976; Hershberger, 1970; Hess, 1950; Ramachandran, 1988). On the basis of this expectation, the surface regions depicted in lighter areas of an image should be perceived as facing upward toward the overhead light source, whereas those depicted in darker areas of the image should be perceived as facing downward (see Figure 2). The global reversal of light and dark regions that occurs when an image is turned upside down thus requires a perceived inversion in depth in order to remain consistent with the assumption of overhead illumination.

One of the clearest demonstrations of an overhead illumination bias in the perception of surface relief has been provided in a recent series of experiments by Berbaum et al. $(1983,1984)$. They asked observers to indicate whether a visually depicted "muffin pan" surface appeared concave or convex under a variety of different illumination conditions. When the direction of illumination was varied horizontally to the left or right of the point of observation in the absence of other information, the perceived relief of the surface was perceptually ambiguous. If, on the other hand, the direction of illumination was varied vertically above or below the point of observation, then the surface relief was almost always perceived unambiguously in the direction that was consistent with an overhead light source.

Although these and other related findings would seem to provide strong evidence that an overhead illumination bias may help to distinguish bumps from dents in at least some contexts, our own experience in studying the perception of smoothly curved surfaces (see Mingolla \& Todd, 1984, 1986; Todd \& Akerstrom, 1987; Todd \& Mingolla, 1983, 1984; Todd \& Reichel, 1989) has led us to conclude that this bias is probably of only marginal significance in natural vision and that it is almost certainly not responsible for the perceptual inversion effect observed in Figure 1. Our reasons for this conclusion are twofold. First, in generating computer simulations of shaded surfaces, we have observed repeatedly that perceived relief remains surprisingly invariant over changes 

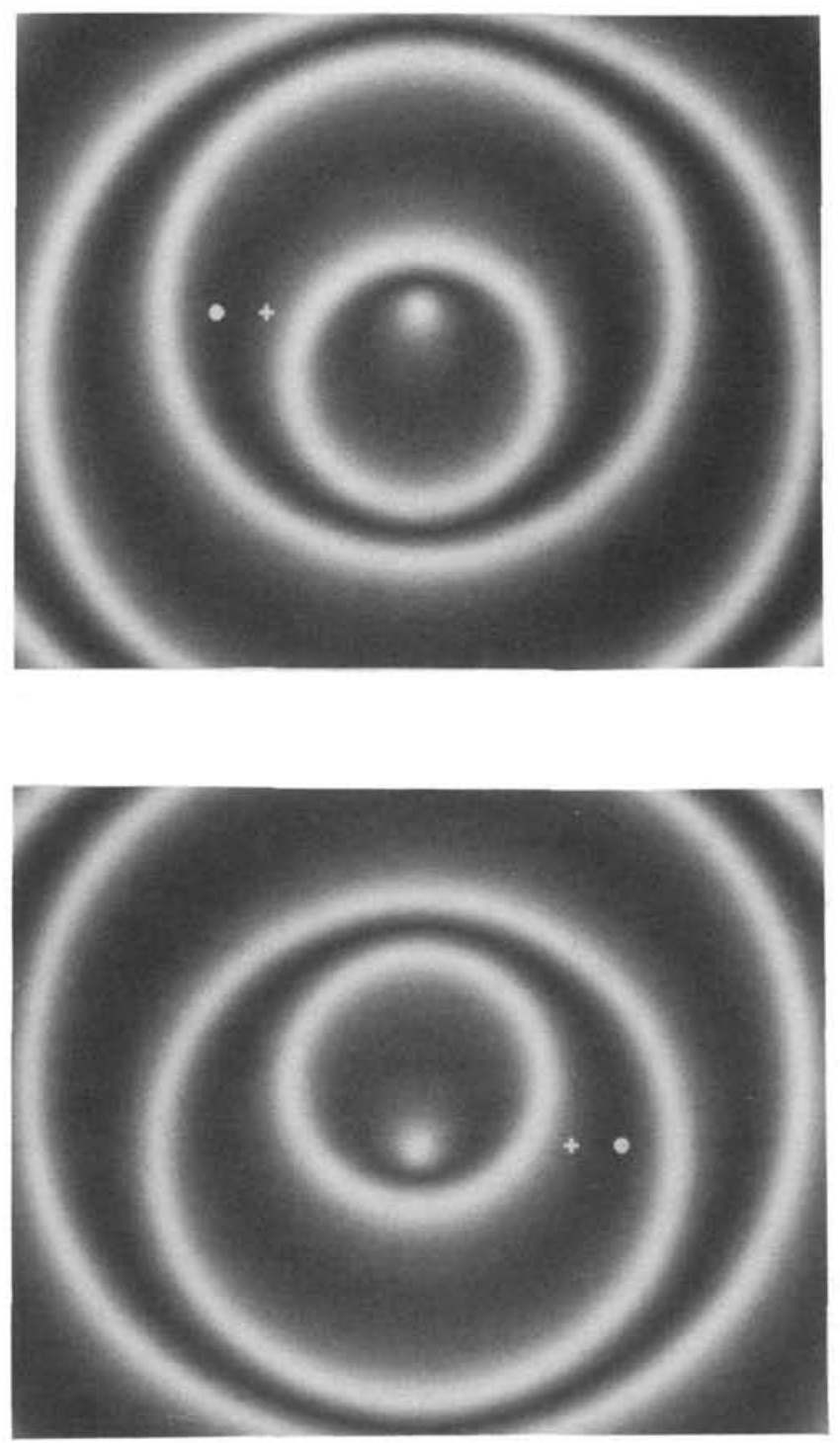

Figure 1. Shaded image of a smoothly curved surface in two different orientations. (Most observers report that the upper surface appears to have a convex protuberance at its center, whereas the lower surface appears to have a concave depression. This perceptual inversion can be measured locally with a relative depth judgment task. Note in the upper figure that the region marked by a dot appears closer in depth than does the neighboring region marked by a cross. When the image is turned upside down, as in the lower figure, the perceived depth order is reversed so that the region marked by the cross appears closer.)

in illumination similar to those used by Berbaum et al. (1984). Consider, for example, the pair of images presented in Figure 3. The depicted surface in this figure is identical in all respects to the one shown in Figure 1, except that the simulated direction of illumination is perpendicular to the image plane. Although an overhead illumination bias would be expected to have no effect in this condition, the perceived relief of the surface reverses with image orientation in exactly the same way as is observed in Figure 1.
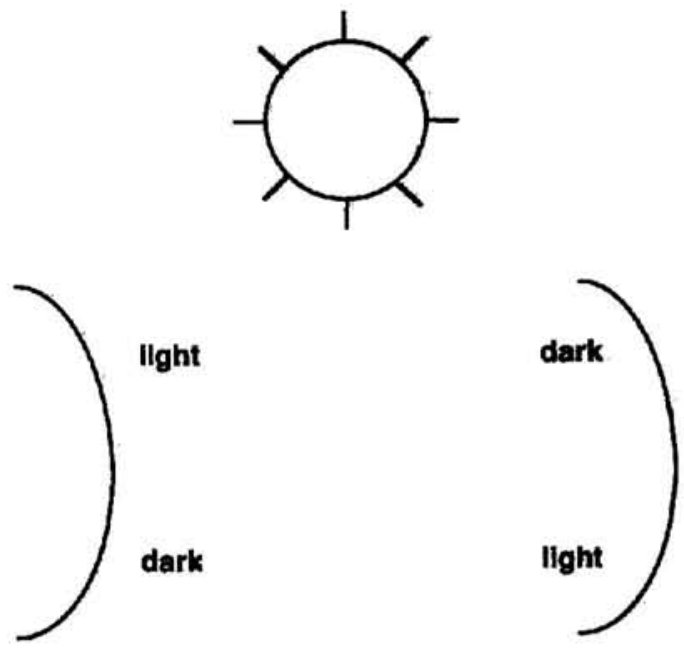

Figure 2. When a surface is illuminated from above, the regions facing upward toward the overhead light source will have a higher luminance than those facing downward, and the direction of the luminance gradient will be uniquely determined by the surface relief. (For a convex surface the luminance gradient will increase in an upward direction, and for a concave surface it will increase in a downward direction. These relationships are reversed when a surface is illuminated from below.)

Second, we have also observed the same type of inversion effect when surfaces are depicted with other forms of pictorial information, such as the pattern of image contours presented in Figure 4 (see also Beusmans, Hoffman, \& Bennett, 1987; Hoffman \& Richards, 1984). Although there is some degree of shading in this pattern because of the variation in contour spacing, it has the opposite contrast in relation to the perceived relief from what would be expected based on an assumption of overhead illumination. Thus, there appears to be some other factor in addition to an overhead illumination bias that is capable of producing a perceived reversal in depth when an image is turned upside down.

To provide a more methodologically rigorous demonstration of these informal observations, we developed a relative local depth judgment task that allows us to obtain a precise measure of the global consistency and accuracy of the perceived relief of smoothly curved surfaces. To better appreciate the logic of this procedure, it is useful to consider a single pair of surface points that are individually labeled with a dot and a cross (e.g., see Figure 1). Suppose that observers consistently respond that the cross appears closer in depth than the dot when the image is presented upright, but that the dot appears closer in depth than the cross when the image is presented upside down. This type of orientationally dependent response indicates that an up-down reversal of image orientation has somehow produced a perceived depth inversion in the local neighborhood of that particular point pair. The global consistency of this perceived depth inversion can then be determined by obtaining multiple judgments in different regions of the same image. It is also possible to detect spontaneous reversals, such as those observed with a Necker cube, by 

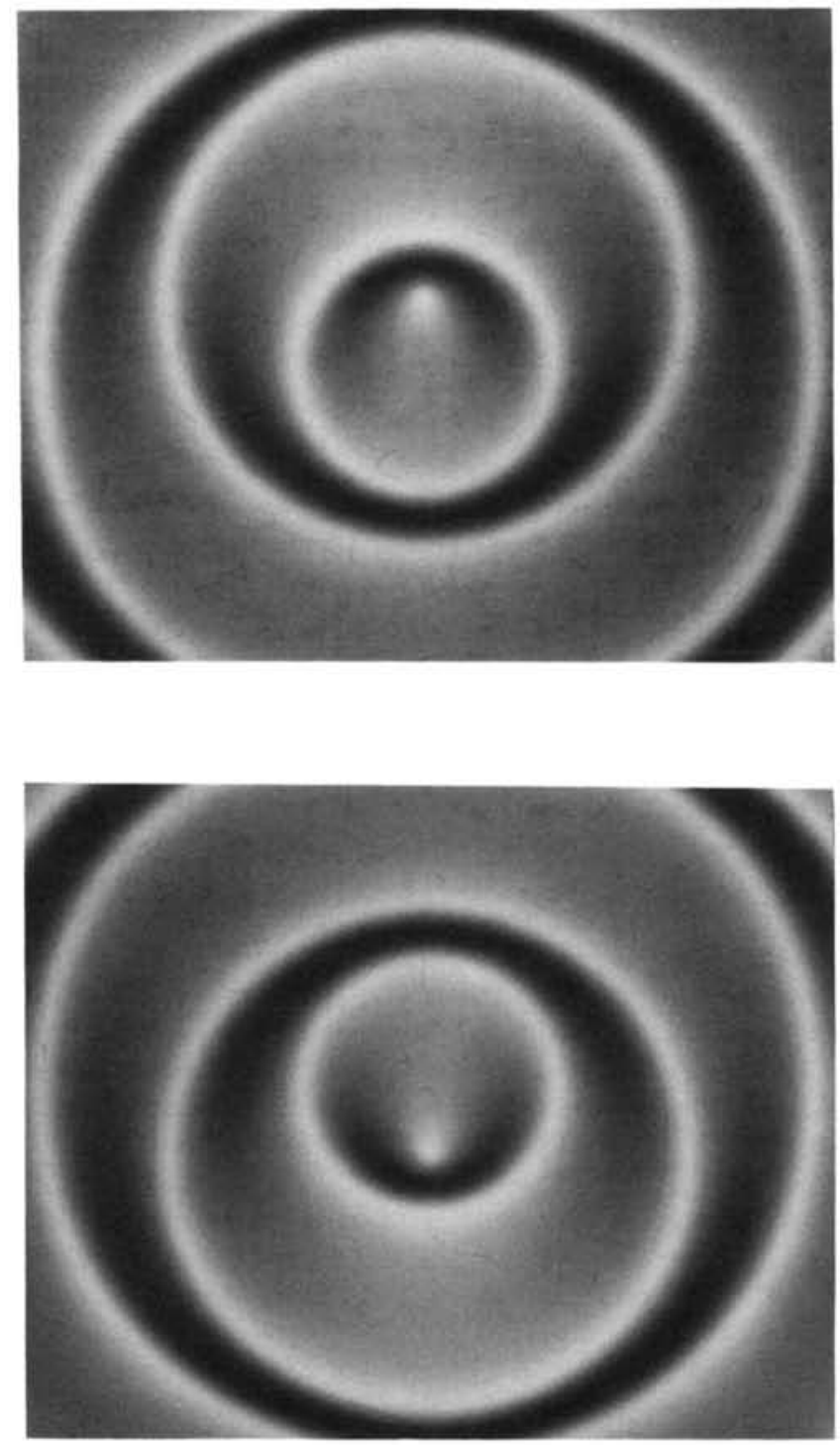

Figure 3. Shaded image of a smoothly curved surface in two different orientations. (The depicted surface is identical to the one shown in Figure 1, except that the simulated light source is positioned at the point of observation.)

obtaining multiple judgments of a given point pair at different moments in time.

In the present series of experiments, we adapted this relative local depth judgment procedure to investigate how a variety of stimulus factors influence perceived depth inversion caused by image orientation. Experiment 1 was designed in particular to compare how this phenomenon is affected by the different types of surface depiction shown in Figures 1, 3, and 4.

\section{Experiment 1}

\section{Method}

Subjects. Six Brandeis University students and staff members volunteered to participate in the experiment.
Apparatus. Stimuli were produced by using a Lex-90 graphics system and were displayed on a 19-in. color monitor. Observers viewed the displays binocularly at a distance of approximately $115 \mathrm{~cm}$. The stimuli were presented within a rectangular region of the display screen that was $33 \mathrm{~cm}$ along the horizontal axis and $26 \mathrm{~cm}$ along the vertical axis. The spatial resolution within this viewing window was $640 \times 512$ pixels. Head movements were not restricted.

Stimuli. Each of the displays was a computer-generated depiction of a smoothly curved shaded surface that filled the entire display screen. The three surfaces shown in Figures 1, 3 , and 4 were presented in two orientations, upright and upside down (i.e., rotated $180^{\circ}$ in the picture plane), for a total of six different displays. The displays were generated with a Cartesian coordinate system $(x, y, z)$, where $x$ and $y$
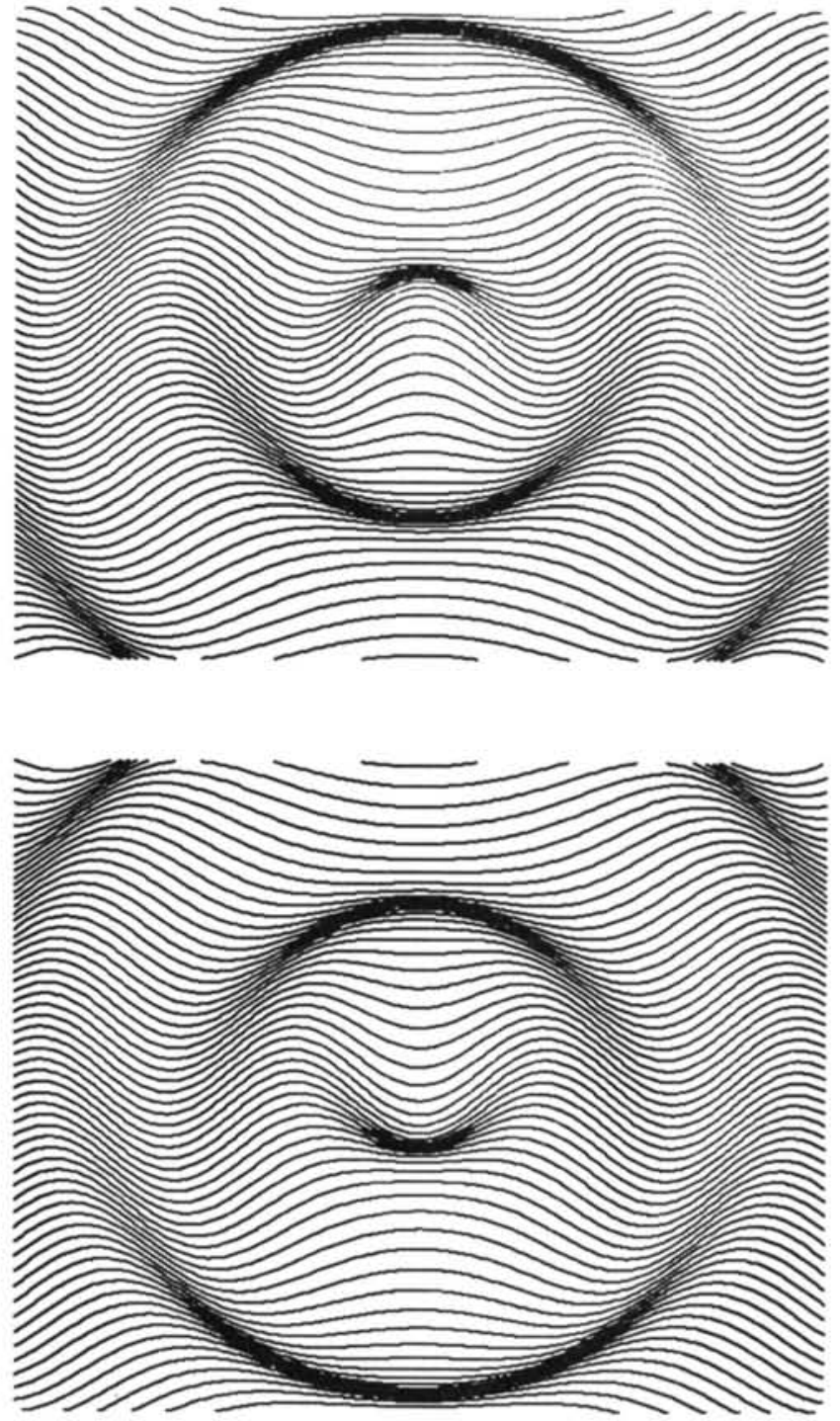

Figure 4. Contoured image of a smoothly curved surface in two different orientations. (The three-dimensional structure of the depicted surface is identical to those shown in Figures 1 and 3.) 
were aligned with the horizontal and vertical axes of the display screen, and $z$ was perpendicular to the screen in depth. The depth $z$ at each point on the surface was defined initially by using the following equation:

$$
z=100 \cos \left(\pi \sqrt{x^{2}+y^{2}} / 100\right) .
$$

It was then rotated $15^{\circ}$ about a horizontal axis through the origin and displayed under parallel projection.

For the two shaded images depicted in Figures 1 and 3, the intensity $(I)$ of each picture element was determined with seven-bit precision by using the following equation:

$$
I=127(\mathbf{L} \cdot \mathbf{N}),
$$

where $\mathbf{L}$ is a unit vector in the direction of the light source, and $\mathbf{N}$ is a unit vector that is perpendicular to the surface at the depicted point (see Todd \& Mingolla, 1983). This relation is a reasonable approximation of how light reflects from a pure matte (Lambertian) surface. For the image shown in Figure 1, the pattern of illumination simulated an infinitely distant point light source at a $20^{\circ}$ angle directly above the point of observation. For the image shown in Figure 3, in contrast, the simulated light source was perpendicular to the display screen to eliminate any possible effects of an overhead illumination bias (cf. Berbaum et al., 1983, 1984).

For the contoured image shown in Figure 4, each individual contour was defined mathematically from Equation 1 by holding $y$ constant at a fixed value $y_{i}$. The pattern of contours was generated by using multiple values of $y_{i}$ in 10 pixel increments. These mathematically defined contours were then rotated $15^{\circ}$ with respect to the initial coordinate system in the plane of the display screen. They were presented under parallel projection as black lines against a white background.

Thirty horizontally separated pairs of points to be judged for relative depth were selected at random so that (a) none of them crossed a local maximum or minimum of depth (see Todd \& Reichel, 1989); (b) they were all separated in depth by a minimum of 11 pixels; and (c) they were evenly distributed over the depicted surface.

Procedure. Observers made relative depth judgments for each of the 30 pairs of points on each of the six experimental displays (i.e., three styles of depiction and two image orientations, upright and upside down). They were alerted that a trial was about to begin by a countdown against a homogeneous black background. In $1-\mathrm{s}$ intervals, the number 3 was displayed, followed by the number 2 , followed by a single pair of small red dots presented in isolation, which allowed observers time to direct their gaze to the appropriate region of the display screen. After this countdown, the two dots remained in view, but the background was replaced by one of the experimental displays. Observers were instructed to indicate which of the two small dots (left or right) marked the surface region that appeared closer in depth; they were instructed to respond as quickly as possible while still maintaining accuracy by depressing one of two specified keys on a computer keyboard. The surface and the pair of dots remained on the screen until a response was recorded. No feedback about the accuracy of their responses was provided during the experiment.
Each observer made 30 judgments for each display in two separate sessions, for a total of 360 judgments per subject. Before the first session, subjects were given practice trials in which they were allowed to familiarize themselves with the depicted surfaces and with the task. Each session lasted about $20 \mathrm{~min}$.

\section{Results and Discussion}

Table 1 shows the percentage of "correct" responses (i.e., those that were consistent with Equation 1) for each of the three images in both possible orientations. Although the definition of a correct response in this context is somewhat arbitrary, because all of the displays were mathematically ambiguous, the designation is useful in order to distinguish the two possible patterns of surface relief. Note in the table that the observers' responses were highly consistent. That is, the observers were almost perfectly accurate when the images were presented upright and almost perfectly inaccurate when the images were presented upside down. This dramatic effect of image orientation was confirmed statistically with an analysis of variance, $F(1,5)=4921.35, p<.01$, accounting for over $99 \%$ of the total variance. There were no significant effects of depiction and no significant differences between the first and second experimental sessions. These findings indicate that the perceived relief was completely determined by image orientation for all three methods of depiction and that there was no effect at all of the simulated direction of illumination.

As was described in the introduction, the image shown in Figure 3 was designed specifically to neutralize any possible effects of an overhead illumination bias by positioning the simulated light source at the point of observation. This logic assumes, however, that the simulated direction of illumination was perceived correctly. If that was not the case, then the observers' judgments might still have been affected by an overhead illumination bias. To test for this possibility, we asked the observers at the conclusion of the experiment to estimate the direction of illumination for the upper image of Figure 3 by using a schematic diagram of the display screen with different illumination directions marked along a circular arc in $5^{\circ}$ increments. As expected, the observers' responses were completely inconsistent with an overhead illumination bias. Indeed, on the basis of the average of their direction judgments, the surface appeared to be illuminated from below at an angle of $33^{\circ}$.

A similar pattern of results in direct contradiction with an overhead illumination bias was also obtained for the contoured image presented in Figure 4 . Note in the figure that

Table 1

Percentage of Correct Responses in Conditions of Experiment I

\begin{tabular}{lccc}
\hline & \multicolumn{2}{c}{ Shading } & \\
\cline { 2 - 3 } Condition & $\begin{array}{c}\text { Overhead } \\
\text { illumination }\end{array}$ & $\begin{array}{c}\text { Eye level } \\
\text { illumination }\end{array}$ & Contours \\
\hline Image upright & 99 & 99 & 99 \\
Image upside down & 2 & 6 & 0 \\
\hline
\end{tabular}


there is some degree of shading due to variable contour spacing but that the perceived relief as indicated by the observers' responses is exactly the opposite of what would be expected based on an assumption of overhead illumination, in that the darker areas of the surface appear to face upward and the lighter areas appear to face downward. In our own informal observations, we have also examined this same display with white contours against a black background. Although this produces a global reversal of image contrast relative to Figure 4, it has no effect whatsoever on the perceived relief of the depicted surface. These observations suggest strongly that the specific orientations of light and dark regions have little to do with the perceived surface relief in these displays.

Why, then, do the surfaces appear to invert in depth when the displays are turned upside down? One possible explanation for this phenomenon is suggested by the classical depth cue of height in field, which is based on the principle that distances of objects from the point of observation tend to increase with their height in the visual field. If we extend this principle to smoothly curved surfaces, it is reasonable to suppose that observers may be biased to perceive surfaces slanted backward (rather than forward) in depth (see Figure 5; see also the similar suggestions of Beusmans et al., 1987; Hoffman \& Richards, 1984; Stevens, 1986). Note that this hypothesis is considerably more general than traditional accounts based on an overhead illumination bias, which are only applicable to certain types of shaded images. If the apparent inversion of smoothly curved surfaces due to image orientation is primarily determined by a perceptual bias for backward slanting surfaces, then the effect should be independent of any particular method of pictorial depiction, just as we observed in the present experiment.

In evaluating alternative factors that can influence the perceptual appearance of these displays, we should also consider the possible effects of an observer's intentions, such as those that have been demonstrated for other multistable stimuli (e.g., see Hochberg \& Peterson, 1987; Peterson \& Hochberg, 1983). Although some experienced observers are indeed capable of making these surfaces reverse in depth through

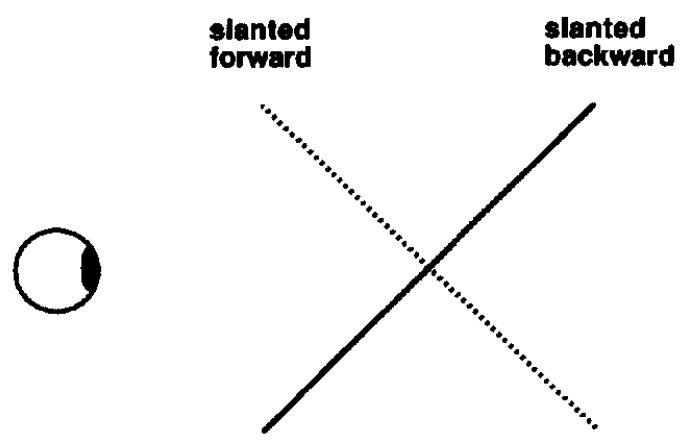

Figure 5. A surface depicted under parallel projection can have two possible orientations in depth, which are mathematically ambiguous. (The results of the present experiments suggest that observers are biased to perceive backward slanting orientations so that perceived distance increases with height in the visual field.) conscious manipulations of attention, it is important to keep in mind that the procedure used in the present experiment was designed specifically to minimize these influences. The observers were not informed that the displays could have multiple interpretations, and they were instructed to respond as quickly as possible after the onset of each display to ensure that the responses would all be based on their immediate first impressions. It seems clear from the data that all of the observers performed as instructed. The average response time was $595 \mathrm{~ms}$ from stimulus onset, with no significant variations among the different conditions. None of the observers reported during debriefing that they had attempted to consciously manipulate their perceptions, and several of them expressed surprise that the depicted surfaces were in any way ambiguous.

\section{Experiment 2}

In all of the images presented thus far, the perceptual inversion in depth due to image orientation is highly consistent over the entire depicted surface. There are many other displays, however, for which this is not the case. Consider, for example, the image shown in Figure 6. The surface depicted in this image is structurally quite similar to the one shown in Figure 1, but it has greater slant in relation to the picture plane so that some parts of the surface are occluded by others. Note in particular the two points marked by a dot and a cross that straddle an occlusion contour. Unlike the surface in Figure 1, the relative positions of these points do not appear to invert in depth when the image is turned upside down; that is, the region marked by a dot appears closer to the observer than the horizontally adjacent region marked by a cross, regardless of image orientation.

It is also important to note, however, that the presence of occlusion contours in Figure 6 does not eliminate the potential for perceptual inversion in all regions of the image. Consider, for example, the other dot and cross that do not straddle an occlusion contour. Many observers report that the surface relief in this region is multistable when the image is turned upside down. If the region marked by a dot appears closer in depth, then the central cone will be perceived as a cone. If, on the other hand, the cross appears closer, then the central cone will be perceived as a flap. It appears from this example that perceptual inversion due to image orientation need not be an all-or-none phenomenon. Some local regions may appear to invert in depth, whereas others remain perceptually stable (cf. Hochberg \& Peterson, 1987; Peterson \& Hochberg, 1983). Experiment 2 was designed to investigate this phenomenon in greater detail.

\section{Method}

The apparatus and procedure were identical in all respects to those used in Experiment 1. The stimuli depicted three different surfaces, each of which could be displayed with either contours or shading. Surface 1 was identical to the one used in Experiment 1. The shaded image of this surface is shown in Figure 3 and the contoured image 


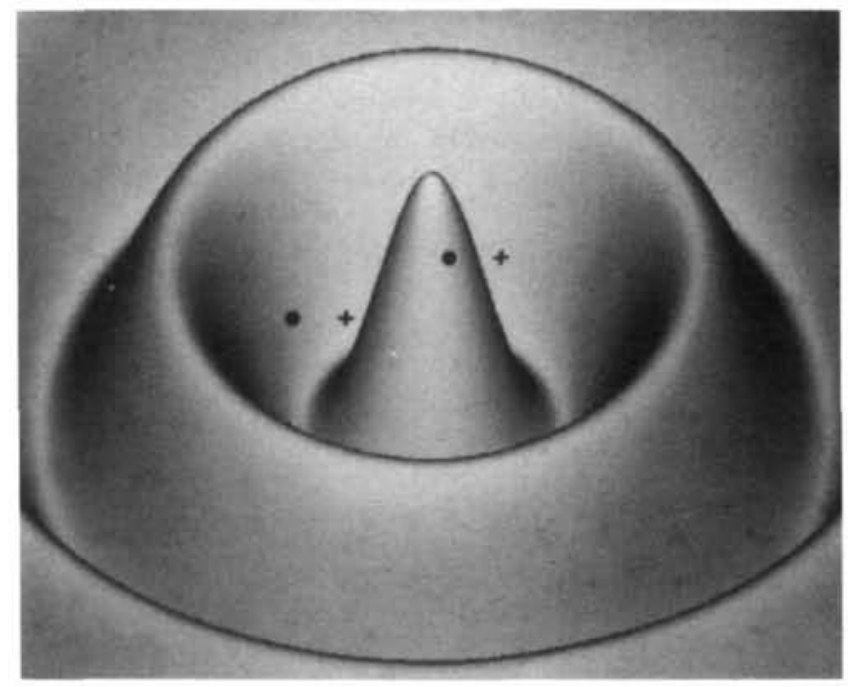

in Figures 6-9 in both upright and inverted orientations. For all of the shaded displays, the simulated direction of illumination was perpendicular to the image plane.

Thirty horizontally separated pairs of points to be judged for relative depth were selected on each of the experimental surfaces. The pairs were distributed over each of the surfaces so that (a) none of them crossed a local maximum or minimum of depth; (b) for Surfaces 2 and 3 , which contain occlusion contours, 10 of the point pairs were chosen so that they straddled an occlusion contour, and the remaining 20 were on smooth surface regions; and (c) point pairs over occlusions were between 150 and 250 pixels apart in depth and point pairs on smooth surface regions were between 11 and 175 pixels apart in depth.

Eight observers judged each of the point pairs on the different displays twice each in both of the possible image orientations for a total of 720 judgments per observer. The experiment was performed over four separate sessions: two with the shaded displays followed by two with the contoured displays. Each session lasted about $20 \mathrm{~min}$.
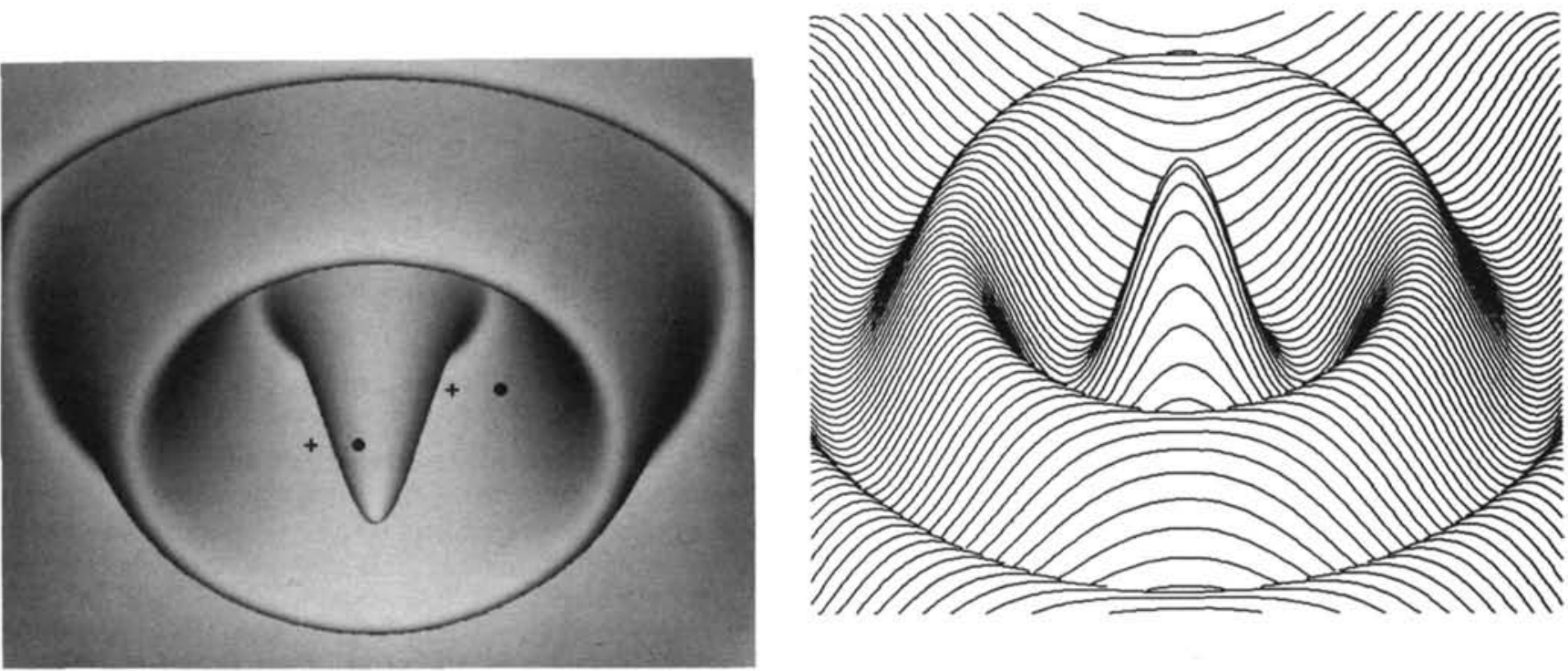

Figure 6. Shaded image of Surface 2 in two different orientations. (Many observers report that this surface does not appear to invert in a globally consistent manner. Consider, for example, the pair of points marked by a dot and cross that straddle an occlusion contour. The region marked by a dot appears closer in depth than does the neighboring region marked by a cross, regardless of image orientation. That is not always the case, however, for the other dot and cross that do not straddle an occlusion. Although the region marked by a dot appears closer in the upper figure, its relative depth can appear multistable when the image is turned upside down.)

is shown in Figure 4. Surfaces 2 and 3 were defined initially by using the following equations:

$$
z=100 \cos \left(\pi \sqrt{x^{2}+y^{2}} / 100\right)+100 e^{-\sqrt{x^{2}+y^{2}} /(800 \pi)}
$$

for Surface 2, and

$$
z=25 \cos (\pi y / 75+\pi / 2)-75 \cos \left(\pi \sqrt{x^{2}+y^{2}} / 125\right)
$$

for Surface 3. They were then rotated $40^{\circ}$ about a horizontal axis through the origin and displayed under parallel projection. The resulting images depicted with either contours or shading are shown

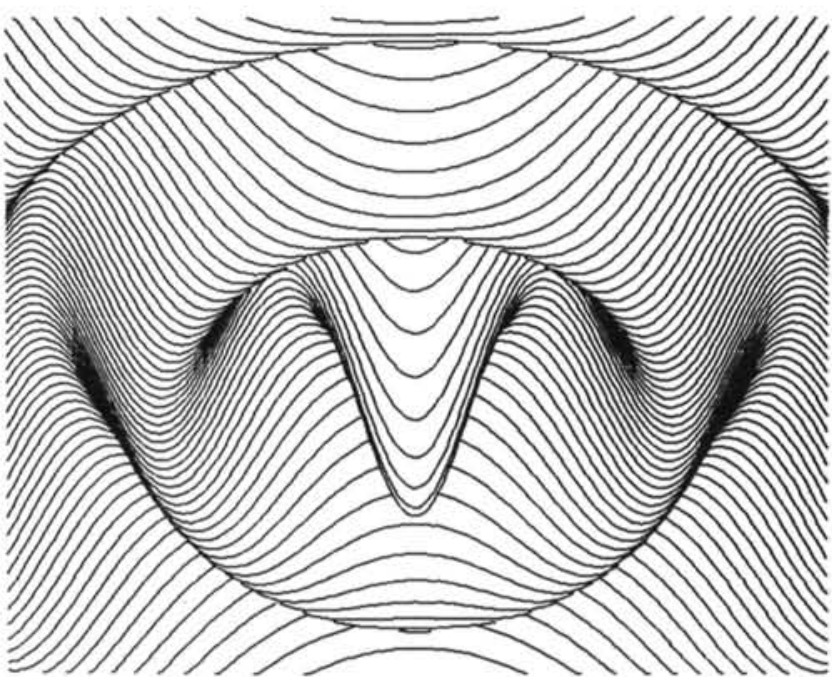

Figure 7. Contoured image of Surface 2 in two different orientations. 

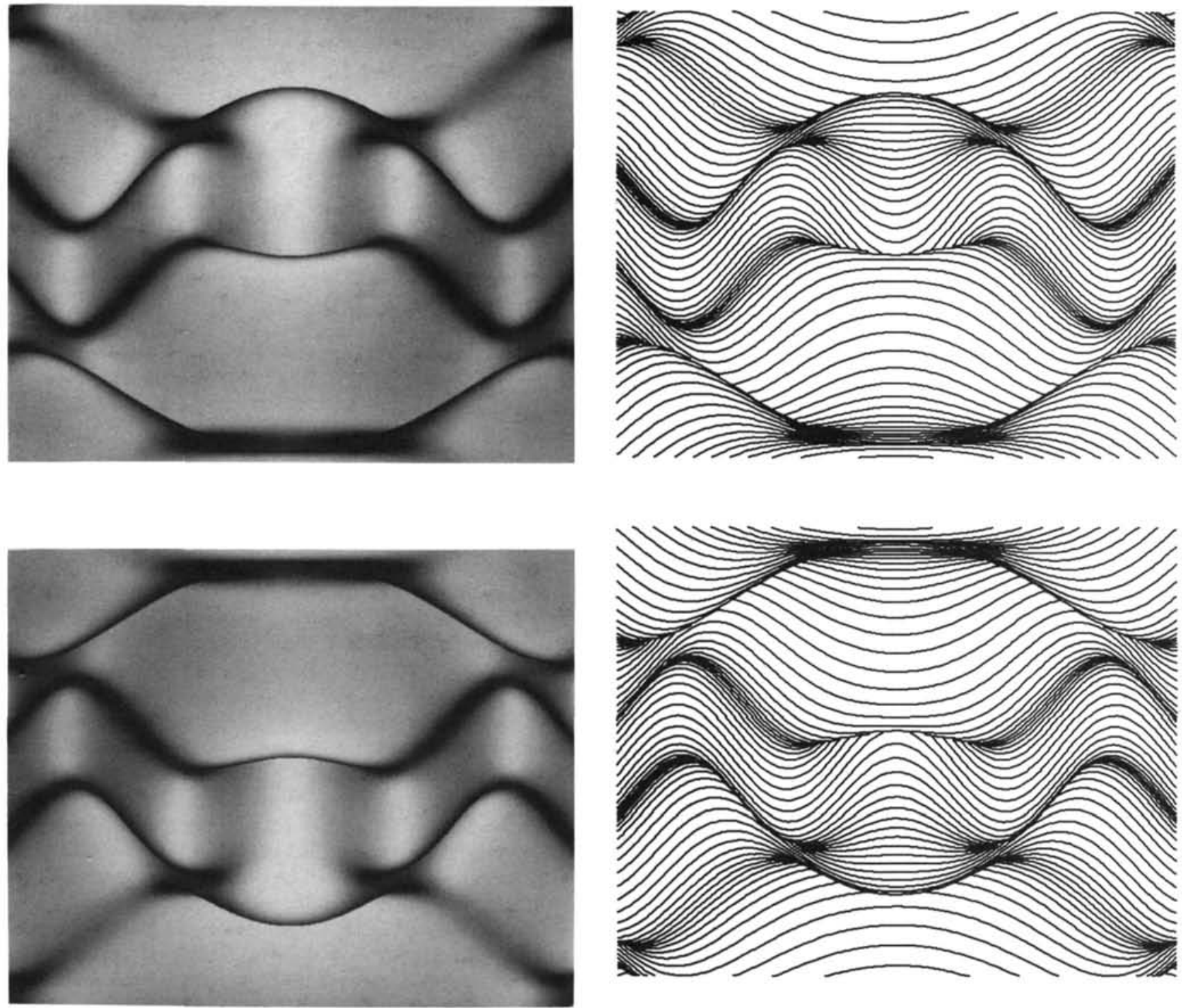

Figure 8. Shaded image of Surface 3 in two different orientations.

Figure 9. Contoured image of Surface 3 in two different orientations.

interaction between the different surfaces and image orientations, $F(2,14)=25.98, p<.001$. As in Experiment 1, there were no significant differences between the shaded and contoured displays and no significant variations in performance over the four experimental sessions.

One possible explanation for the intermediate levels of accuracy with the inverted images of Surfaces 2 and 3 is that

observers' responses to Surface 1 were highly consistent, jus as they were in the previous experiment. The responses were highly accurate when the images were presented upright and highly inaccurate when the images were presented upside down. Surfaces 2 and 3 , in contrast, produced a very different pattern of results. The observers were highly accurate when the images were presented upright, but they exhibited an intermediate level of performance when the images were turned upside down.

These observations were confirmed statistically with an analysis of variance. The analysis revealed a significant effect of image orientation, $F(1,7)=122.78, p<.001$; a significant effect of surfaces, $F(2,14)=21.64, p<.001$; and a significant

Table 2

Percentage of Correct Responses in Conditions of Experiment 2

\begin{tabular}{lccc}
\hline Condition & Surface 1 & Surface 2 & Surface 3 \\
\hline Shading & & & \\
Image upright & 97 & 99 & 86 \\
Image upside down & 12 & 78 & 51 \\
Contours & 96 & 98 & 93 \\
Image upright & 6 & 53 & 39 \\
Image upside down & & & \\
\hline
\end{tabular}


these displays are perceptually multistable. Suppose, for example, that a depicted surface appears to invert spontaneously in depth over time, in much the same way as a Necker cube. The perceived relief in that case would be expected to change from trial to trial, producing intermediate levels of both accuracy and test-retest reliability.

However, another possible explanation for this result that is more consistent with the observers' subjective impressions is that the images of Surfaces 2 and 3 are perceived to undergo a piecemeal reversal when they are turned upside down, so that some local regions reliably appear to reverse in depth whereas others remain reliably stable. This type of piecemeal reversal would also be expected to produce intermediate levels of accuracy, but in contrast to the effects of spontaneous reversal, there should be a high level of test-retest reliability for individual point pairs within a given orientation.

In an effort to provide some empirical evidence to distinguish these hypotheses, we computed the test-retest reliabilities for each point pair on each surface both within and between image orientations. The results are presented in Table 3. Note in the table that the observers' judgments were highly reliable within a given image orientation. This result shows clearly that all of the displays had a high degree of withinorientation stability and that the perceived depth orders of individual point pairs seldom inverted spontaneously over successive trials. When considered in combination with the intermediate levels of reliability between orientations, these findings provide strong support for the observers' subjective impressions that the surfaces appeared to invert in a piecemeal fashion when the images were turned upside down. This result is also consistent with similar patterns of piecemeal reversal that have been reported previously for Necker cube stimuli by Peterson and Hochberg (1983) and Hochberg and Peterson (1987).

One possible source of variation in these stimuli that is likely to be responsible for their varying susceptibility to perceptual inversion is the presence or absence of occlusion contours (cf. Howard, 1982). Note in particular that the images of Surfaces 2 and 3 with visible occlusions appeared to invert in a piecemeal fashion, whereas the images of Surface

Table 3

Test-Retest Reliability of Individual Point Pairs for Conditions of Experiment 2 Both Within and Between Image Orientations

\begin{tabular}{cccc}
\hline Condition & Surface 1 & Surface 2 & Surface 3 \\
\hline $\begin{array}{l}\text { Shading } \\
\text { Within orientation, }\end{array}$ & .95 & .99 & .93 \\
$\begin{array}{l}\text { image upright } \\
\text { Within orientation, }\end{array}$ & .86 & .93 & .87 \\
$\quad \begin{array}{l}\text { image upside down } \\
\text { Between orientations }\end{array}$ & .17 & .78 & .48 \\
$\begin{array}{l}\text { Contours } \\
\text { Within orientation, }\end{array}$ & .92 & .96 & .89 \\
$\quad \begin{array}{l}\text { image upright } \\
\text { Within orientation, }\end{array}$ & .90 & .89 & .87 \\
$\quad \begin{array}{l}\text { image upside down } \\
\text { Between orientations }\end{array}$ & .10 & .53 & .40 \\
\hline
\end{tabular}
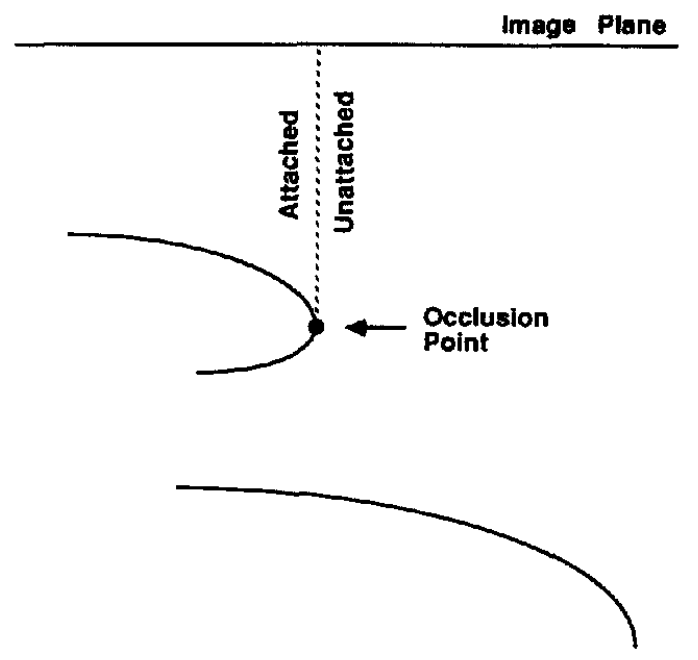

Figure 10. An occlusion point separates a visible scene into two distinct regions, one that is attached to the occlusion and another that is unattached. (Identifying an occlusion point with a correct labeling of its attached and unattached regions places two important constraints on the surface relief. First, the surface depth must decrease monotonically as one moves from an occlusion point in an attached region, and second, the attached region must be closer in depth than the unattached region.)

1 with no visible occlusions were perceived to invert completely. To better appreciate why this should be the case, it is important to recognize that an occlusion contour separates a visible scene into two distinct regions: one that is attached to the occlusion and another that is unattached (see Figure 10). The correct identification of an occlusion contour, with appropriate labeling of its attached and unattached regions, places two fundamental restrictions on the possible surface relief in the neighborhood of the occlusion: First, as we move from an occlusion contour in an attached region, the depth of the surface must decrease monotonically until a depth minimum is reached (see Todd \& Reichel, 1989); and second, the visible surface on the attached side of an occlusion must always be closer in depth than its corresponding region on the unattached side.

It is also important to point out, however, that the presence of occlusion contours in an image does not necessarily guarantee that observers will correctly identify their attached and unattached sides. To assess the accuracy of this labeling in the present experiment, we performed a separate analysis to examine the perceived depth order relations of those point pairs that straddled an occlusion contour. The analysis revealed that observers were $98 \%$ accurate for these point pairs when the images were presented upright, but the accuracy was reduced to only $62 \%$ when the images were presented upside down. This finding demonstrates that (a) some occlusion contours provide inadequate information to perceptually distinguish their attached and unattached regions, and (b) these occlusions are therefore incapable of inhibiting perceptual inversion in their immediate local neighborhoods when an image is turned upside down. 
To summarize, the results of Experiment 2 suggest two interacting mechanisms that can influence the perceived relief of smoothly curved surfaces. We believe that some information about surface relief in individual local neighborhoods is provided by occlusion contours but that there is also an overall bias to perceive a surface slanting backward rather than forward (see Figure 5). If both of these constraints are in agreement with each other (as in the upright images of Figures 6-9) or if there are no visible occlusion contours (as in Figures 1,3 , and 4), then observers' relative depth judgments for a given surface should be globally consistent. If, on the other hand, the two constraints are incompatible (as in the upside down images of Figures 6-9), then the perceived relief may be globally inconsistent and the depicted surface may appear phenomenally similar to an impossible figure.

\section{Experiment 3}

An important implication of the preceding analysis is that perceived depth inversions of smoothly curved surfaces caused by changes in image orientation should not be dependent on the particular source of optical information through which a surface is pictorially represented. If, as we argued earlier, the inversion effects of Experiments 1 and 2 are due primarily to a perceptual bias for backward slanting surfaces, then the same effects should also be observable with other sources of information besides shading or contours. Experiment 3 was designed specifically to test this prediction by using surfaces specified by optical motion.

\section{Method}

The simulated surfaces were identical to those used in Experiment 2 , but their pictorial representation was quite different. On each trial, observers were presented with an apparent motion sequence consisting of seven distinct images that cycled back and forth in rapid succession, with a stimulus onset asynchrony of $83 \mathrm{~ms}$ and an interstimulus interval of zero. In the initial frames of these sequences, the orientations of the depicted surfaces were identical to those described in Experiment 2. The remaining frames were created by rotating the surfaces forward about a horizontal axis in $1.5^{\circ}$ increments for each frame transition. After an entire sequence of seven frames was completed, it was then repeated in the opposite direction, which appeared to the observers as a smoothly curved surface in continuous oscillation.

Each frame of the apparent motion sequences contained 327,680 distinct picture elements, each of which had a $50 \%$ probability of being black or white (see Figure 11). That is, each individual image contained a homogeneous pattern of random noise, with no information whatsoever from shading or texture that could potentially specify the structure of the depicted surface. This constraint was achieved by adding or subtracting noise elements as needed to compensate for the projective expansion and contraction of each surface region during the course of its rotation in depth (cf. Sperling, Landy, Dosher, \& Perkins, 1989).

Because the relative depths of individual surface regions varied over time as a result of the apparent motion, it was necessary to alter slightly the procedure used in the two previous experiments. During each trial, a single pair of small red dots was superimposed over the random noise in the initial frame of the apparent motion sequence. The dots were removed for Frames 2-7, but they reappeared again
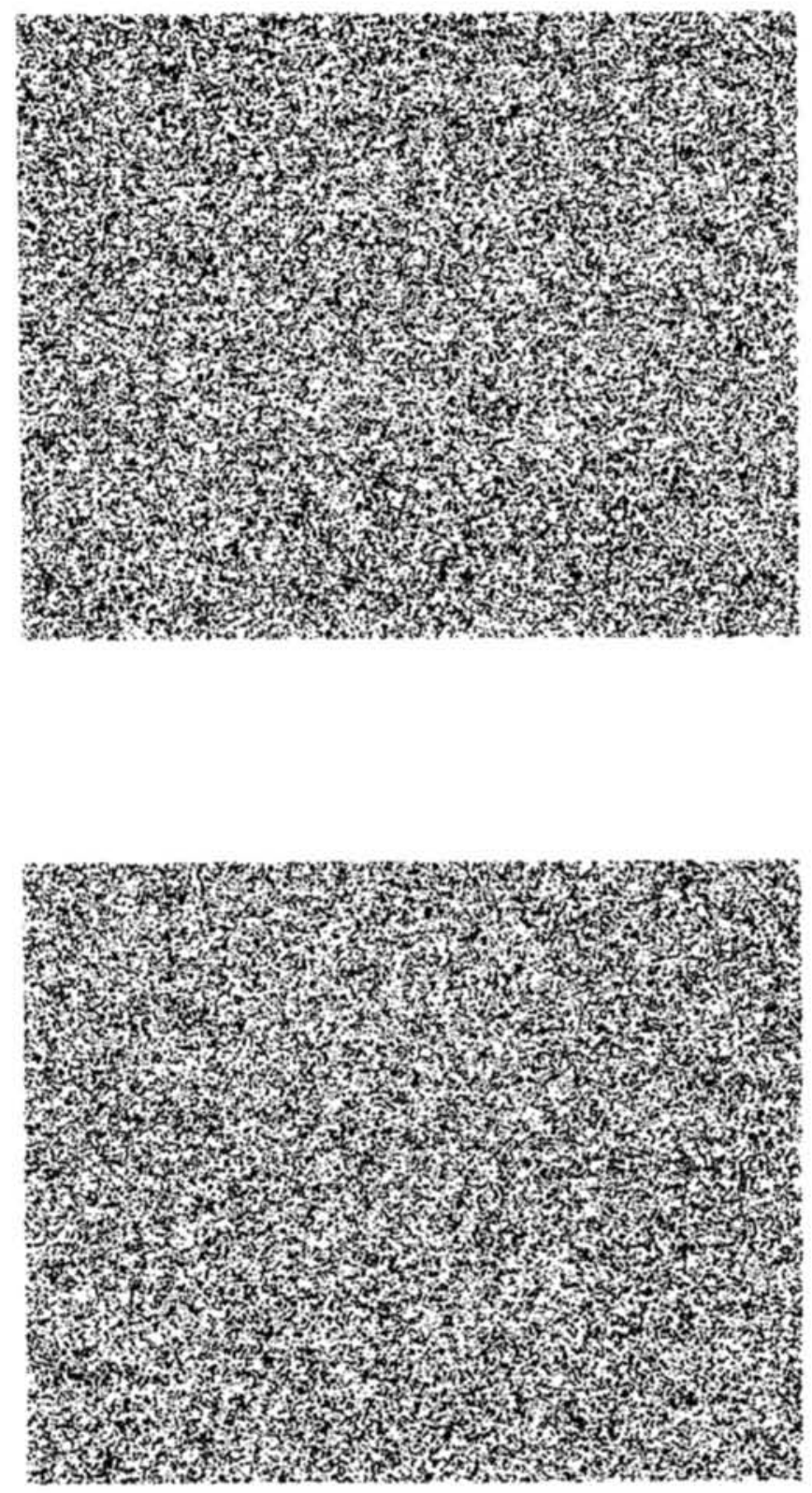

Figure 11. Two patterns of homogeneous random noise similar to those used in Experiment 3 as part of the apparent motion sequences. (The depicted surface can be observed by turning the page sideways and viewing the patterns stereoscopically.)

each time the sequence cycled back to Frame 1. Observers were instructed to indicate which of the two small dots (left or right) marked the surface region that appeared closest in depth during the brief periods of time when the dots were visible. They were instructed to respond as soon as they were confident of the depicted depth order by depressing one of two specified keys on the computer keyboard. The surface appeared in continuous oscillation with the two red dots blinking on and off until an appropriate response was recorded.

Six observers made relative depth judgments for the same 30 point pairs on each surface that were used in Experiment 2 . The individual point pairs were judged twice each in both possible image orientations for a total of 360 judgments per observer. The experiment was 
performed over two separate sessions, each of which lasted about 20 min.

\section{Results and Discussion}

Table 4 shows the percentage of "correct" responses for each of the three displays in both possible orientations. Note in the table that the observers' responses were highly consistent. Performance was almost perfectly accurate when the displays were presented upright and almost perfectly inaccurate when the displays were presented upside down.

These results strongly support our general hypothesis that a perceptual bias for backward slanting surfaces is a primary determinant of why images of smoothly curved surfaces appear to invert in depth when they are turned upside down. The displays used in this experiment were designed specifically so that observers' judgments could not be influenced-even in principle-by perceptual biases concerning possible patterns of surface texture or illumination. To eliminate all possible sources of information from shading or texture, we ensured that each individual frame of the apparent motion sequences contained a homogeneous pattern of random noise, in which each individual pixel had a $50 \%$ probability of being black or white. It should alsa be kept in mind, moreover, that the displays were generated by using parallel projection so that the depicted relief would be mathematically ambiguous. Nevertheless, from the standpoint of the observers' perceptions, there was no ambiguity whatsoever. Each individual display almost always appeared as a backward slanting surface (see Figure 5), with remarkable stability over hundreds of multiple presentations.

It is also interesting to note in this regard that the presence or absence of occlusion contours in these displays had no effect on the observers' judgments, as they did in Experiment 2 . We believe this is due to an absence of information necessary to identify the attached and unattached sides of an occlusion. To better appreciate our theoretical motivation for this hypothesis, it is important to distinguish between the selfocclusions of smoothly curved surfaces and the occlusions that occur at sharp edges. Consider, for example, a flat piece of paper with sharp edges that is moved over a textured background. The accretion or deletion of texture in that case is only observed on the unattached side of the occlusion and can therefore be used as a potential source of information for determining the relative depth orders of the observed surfaces (see Kaplan, 1969). This information is less useful, however, for the self-occlusions of smoothly curved surfaces, such as those that were observed in Experiment 3 (cf. Todd, 1985). Accretion or deletion of texture in this case can occur on either side of an occlusion and cannot therefore provide

Table 4

Percentage of Correct Responses in Conditions of Experiment 3

\begin{tabular}{cccc}
\hline Condition & Surface 1 & Surface 2 & Surface 3 \\
\hline Motion & & & \\
Image upright & 99 & 96 & 99 \\
Image upside down & 4 & 3 & 3 \\
\hline
\end{tabular}

information for constraining the surface relief in its immediate local neighborhood.

\section{General Discussion}

Some of the most compelling phenomena of visual perception involve multistable stimuli that can appear phenomenally to reverse in depth. For purposes of the present discussion, it is useful to distinguish two different types of perceptual inversion: (a) spontaneous reversals for a fixed pattern of visual stimulation, in which the perceived relief of an object appears to change suddenly from one moment to the next; and (b) reversals due to image orientation, in which objects depicted in a fixed visual image appear stable over time but are perceived to reverse in depth when the image (or the observer) is turned upside down.

In the present series of experiments, we have attempted to distinguish these two types of reversal within individual local neighborhoods of smoothly curved surfaces. To determine the perceived local relief of a surface, observers were required to judge the relative depths of neighboring surface points, and they were instructed to respond as quickly as possible from the onset of each display so that the judgments would all be based on their immediate first impressions. The frequency of spontaneous reversals for these stimuli was quite low; that is, there was a high test-retest reliability for the perceived depths of individual point pairs within a fixed visual image. When the images were turned upside down, however, the depicted surfaces often appeared to invert in depth, as was revealed statistically by a relatively low test-retest reliability for individual point pairs in different orientations.

The perceived depth inversion of smoothly curved surfaces due to image orientation is a phenomenon familiar to most perceptual psychologists. Indeed, it was first reported by Rittenhouse (1786) over 200 years ago and was described in considerable detail by Brewster in 1826. As we indicated in the introduction, the generally accepted explanation for this phenomenon is that observers are biased to perceive surfaces with overhead illumination so that lighter regions face upward and darker regions face downward (see Figure 2). According to the traditional account, this bias is maintained when an image is turned upside down by observers' perceiving its depicted surfaces with an inverted relief.

Although there is considerable evidence that an overhead illumination bias may influence observers' perceptions in certain contexts (e.g., see Figure 12), it is most unlikely to have played a significant role in the present experiments. Several sources of evidence support this conclusion. For example, when the simulated light source in shaded displays was positioned at the point of observation, the effects of inversion were just as large as when the displays simulated an overhead pattern of illumination. Similar effects were also obtained for surfaces depicted with patterns of contours. Although there was some degree of shading in these displays caused by variable contour spacing, the contrast of this shading was reversed in relation to the perceived relief from what would be expected based on an overhead illumination bias. Finally, we also obtained identical effects when the surfaces were depicted with patterns of image motion in which all 


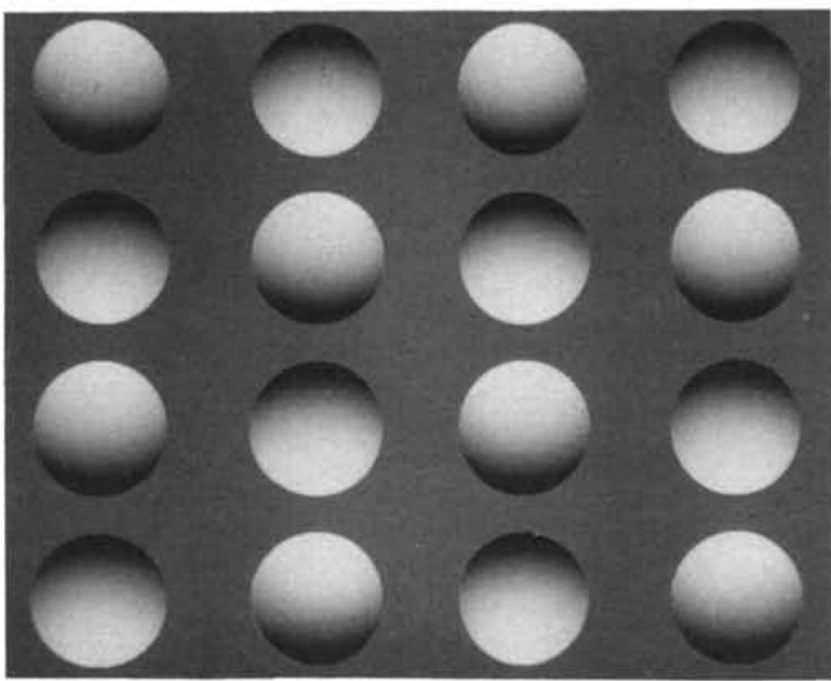

Figure 12. Shaded image of a planar surface that appears to be covered by bumps and dents. (Although the bumps and dents appear perceptually quite different, they all have identical patterns of shading except for their orientations in the image plane. This type of display was first studied by the Finnish psychologist von Fieandt [1938, 1949] over 50 years ago and is similar to those used by numerous other investigators to demonstrate the existence of an overhead illumination bias. Note in particular that a backward slanting surface bias cannot account for the perceived relief in this case because the surface is globally parallel to the image plane with no apparent slant.)

possible information from shading or texture had been eliminated.

One possible alternative explanation of these phenomena is that observers are biased to perceive surfaces with backward slanting orientations so that depth appears to increase with height in the visual field. Because this hypothesis is considerably more general than traditional accounts based on an overhead illumination bias, it is able to provide a plausible explanation for why similar patterns of perceptual inversion are obtained for surfaces depicted with either shading, texture, or motion. We believe it is the case, moreover, that a perceptual bias for backward stanting surfaces can also account for most examples of the so-called "crater illusion" used in many introductory textbooks to demonstrate the existence of an overhead illumination bias. This is an easily testable hypothesis. For any given example of perceptual inversion due to image orientation, it should be possible to place these two different biases in competition with one another by reversing the stimulus contrast (e.g., by using a photographic negative). If the inversion effect is due to an overhead illumination bias, then the negative image should be perceived with inverted relief (see Figure 2). If, on the other hand, the effect is due to a backward slanting surface bias, then the positive and negative images should both be perceived with the same relief.

Although much of the present discussion has focused on the perceptual consequences of a backward slanting surface bias, we do not wish to suggest that this is the only possible factor that can influence the perceived depth inversion of smoothly curved surfaces due to image orientation (e.g., see Figure 12). Another relevant factor that seems to have been important in the present experiments is the presence or absence of occlusion contours in an image. In Experiment 2, for example, the images that contained occlusions were much less susceptible to perceptual inversion than were those that did not contain occlusions.

It is especially interesting to note in this regard that these effects of occlusion were not perfectly reliable. They did not occur at all for the surfaces defined by motion in Experiment 3 , and they were not globally consistent for the surfaces defined by shading or texture in Experiment 2 (cf. Hochberg \& Peterson, 1987; Peterson \& Hochberg, 1983). We strongly suspect, however, that these inconsistent results may not be representative of the effects of occlusion in natural vision. Real visual scenes contain much richer occlusion information than did the relatively impoverished displays used in the present experiments, and we suspect this is why they almost never appear to invert in depth when observed from an upside-down viewing position. For an occlusion contour to constrain the surface relief in its immediate local neighborhood, it must first be identified as an occlusion-as opposed, for example, to a shadow, a specular highlight, or a change in surface reflectance-and then have its attached and unattached regions correctly labeled. Thus, the presence of occlusion contours can only facilitate the perception of surface relief when there is sufficient information available to adequately solve the problem of contour classification. How this problem is actually addressed in natural vision is a fundamental issue that remains for future research.

\section{References}

Benson, C. W., \& Yonas, A. (1973). Development of sensitivity to static pictorial depth information. Perception \& Psychophysics, 13 , 361-366.

Berbaum, K., Bever, T., \& Chung, C. S. (1983). Light source position in the perception of object shape. Perception, 12, 411-416.

Berbaum, K., Bever, T., \& Chung, C. S. (1984). Extending the perception of shape from known to unknown shading. Perception, 13, 479-488.

Beusmans, J. M. H., Hoffman, D. D., \& Bennett, B. M. (1987). Description of solid shape and its inference from occluding contours. Journal of the Optical Society of America A, 4, 1155-1167.

Brewster, D. (1826, January). On the optical illusion of the conversion of cameos into intaglios and of intaglios into cameos, with an account of other analogous phenomeng. Edinburgh Journal of Science, 4, 99-108.

Fieandt, K. von (1938). Uber das Sehen von Tiefengebilden bei wechseinder Beleuchtungsrichtung [Improvements in depth perception by changes in lighting]. Helsinki, Finland: Psychologisches Institut Universitat Helsinki.

Fieandt, K. von (1949). Das phanomenologische Problem von Licht und Schatten [The phenomenological problem of light and shadow]. Acta Psychologica, 6, 337-357.

Gibson, J. J. (1950). The perception of the visual world. Boston: Houghton Mifflin.

Hagen, M. A. (1976). The development of sensitivity to cast and attached shadows in pictures as information for the direction of the source of illumination. Perception \& Psychophysics, 20, 25-28.

Hershberger, W. (1970). Attached-shadow orientation perceived as depth by chickens reared in an environment illuminated from below. Journal of Comparative and Physiological Psychology, 73, 407-411. 
Hess, E. H. (1950). Development of the chick's responses to light and shade cues of depth. Journal of Comparative and Physiological Psychology, 43, 112-122.

Hochberg, J., \& Peterson, M. A. (1987). Piecemeal organization and cognitive components in object perception: Perceptually coupled responses to moving objects. Journal of Experimental Psychology: General. 4, 370-380.

Hoffman, D. D., \& Richards, W. A. (1984). Parts recognition. Cognition, 18, 65-96.

Howard, I. P. (1982). Occluding edges in apparent reversal of convexity and concavity. Perception, 12, 85-86.

Kaplan, G. (1969). Kinetic disruption of optical texture: The perception of depth at an edge. Perception \& Psychophysics, 6, 193-198.

Mingolla, E., \& Todd, J. T. (1984). Computational techniques for the graphic simulation of quadric surfaces. Journal of Experimental Psychology: Human Perception and Performance, 10, 740-745.

Mingolla, E., \& Todd, J. T. (1986). Perception of solid shape from shading. Biological Cybernetics, 53, 137-151.

Peterson, M. A., \& Hochberg, J. (1983). Opposite-set measurement procedure: A quantitative analysis of the role of local cues and intention in form perception. Journal of Experimental Psychology: Human Perception and Performance, 9, 183-193.

Ramachandran, V. S. (1988). Perceiving shape from shading. Scientific American, 252, 76-83.

Rittenhouse, D. (1786). Explanation of an optical deception. Transactions of the American Philosophical Society, 2, 37-42.

Sperling, G., Landy, M. S., Dosher, B. H., \& Perkins, M. E. (1989). Kinetic depth effect and identification of shape. Journal of Exper- imental Psychology: Human Perception and Performance, 15, 826840.

Stevens, K. A. (1986). Inferring shape from contours across surfaces. In A. P. Pentland (Ed.), From pixels to predicates (pp. 93-110). Norwood, NJ: Ablex.

Todd, J. T. (1985). The perception of structure from motion: Is projective correspondence of moving elements a necessary condition? Journal of Experimental Psychology: Human Perception and Performance, 11, 689-710.

Todd, J. T., \& Akerstrom, R. A. (1987). The perception of threedimensional form from patterns of optical texture. Journal of Experimental Psychology: Human Perception and Performance, 13, 242-255.

Todd, J. T., \& Mingolla, E. (1983). The perception of surface curvature and direction of illumination from patterns of shading. Journal of Experimental Psychology: Human Perception and Performance, 9. 583-595.

Todd, J. T., \& Mingolla, E. (1984). The simulation of curved surfaces from patterns of optical texture. Journal of Experimental Psychology: Human Perception and Performance, 10, 734-739.

Todd, J. T., \& Reichel, F. D. (1989). Ordinal structure in the visual perception and cognition of smoothly curved surfaces. Psychological Review, 96, 643-657.

Received August 31, 1988

Revision received July 17,1989

Accepted July 18, 1989 